\title{
Análise das variáveis ambientais em salas cirúrgicas: fontes de contaminação
}

\author{
Analysis of environmental variables in operating rooms: Sources of contamination \\ Análisis de las variables ambientales en quirófanos: fuentes de contaminación
}

Herriét de Araujo Sevilha ${ }^{1}$, Lívia Silva Julião Paiva², Vanessa de Brito Poveda ${ }^{3}$

RESUMO: Objetivo: Analisar as variáveis ambientais que podem contribuir para a ocorrência de infecção do sítio cirúrgico em pacientes submetidos a cirurgias eletivas, em um hospital filantrópico. Método: Trata-se de estudo descritivo, observacional, transversal e com abordagem quantitativa, realizado entre 2011 e 2012. Resultados: Foram analisadas 23 cirurgias de diversas especialidades, com duração média de 54 minutos. As menores médias de temperatura e umidade do ar da sala operatória foram obtidas aos 140 minutos, com $21,3{ }^{\circ} \mathrm{C}$ de temperatura e $29 \%$ de umidade. Houve uma média de 5,69 pessoas em sala, sendo que a média de entradas e saídas foi de 4,34 pessoas. A porta foi aberta, em média, 12,6 vezes, e permaneceu aberta, em média, por 11,89 minutos. A touca, a máscara e o sapato foram utilizados de forma inadequada por parte dos presentes. Conclusão: Evidenciou-se que muitas atividades em sala de operação são realizadas de forma inapropriada, reforçando a necessidade de educação em serviço.

PALAVRAS-CHAVE: Salas cirúrgicas. Infecção hospitalar. Infecção da ferida operatória. Enfermagem.

ABSTRACT: Objective: To analyze the environmental variables that contribute to the occurrence of surgical site infection in patients undergoing elective surgery in a charity hospital. Method: This is a descriptive, observational, cross-sectional study with quantitative approach conducted between 2011 and 2012. Results: Twenty-three surgeries of various specialties, with average duration of 54 minutes, were analyzed. The lowest average air temperature and humidity in the operating room occurred at 140 minutes: $21.3{ }^{\circ} \mathrm{C}$ temperature and $29 \%$ humidity. There was an average of 5.69 people in the room, and the average of entrances and exits was 4.34 people. The door was open 12.6 times and remained open for 11.89 minutes, on average. Caps, masks and shoes were used inadequately by those present in the room. Conclusion: It is evident that many activities in the operating room are performed inappropriately, reinforcing the need for in-service education.

KEYWORDS: Operating rooms. Cross infection. Surgical wound infection. Nursing.

RESUMEN: Objetivo: Analizar las variables ambientales que pueden contribuir a la aparición de la infección del local quirúrgico en pacientes sometidos a cirugía electiva en un hospital filantrópico. Método: Se trata de un estudio descriptivo, observacional, transversal, con abordaje cuantitativo, realizado entre 2011-2012. Resultados: se analizaron 23 cirugías en diversas especialidades, con una duración media de 54 minutos. Las medias de temperatura y humedad más bajas del quirófano se obtuvieron a los 140 minutos: 21,3 grados y $29 \%$ de humedad. Hubo un promedio de 5,69 personas en la sala, y el promedio de entradas y salidas fue de 4,34 personas. La puerta se abrió un promedio de 12,6 veces y permaneció abierta un promedio de 11,89 minutos. El gorro, la máscara y el zapato se utilizaron indebidamente. Conclusión: se evidenció que muchas de las actividades en el quirófano se realizaron de forma inadecuada, lo que refuerza la necesidad de formación continua.

PALABRAS CLAVE: Quirófanos. Infección hospitalaria. Infección de herida operatória. Enfermería.

${ }^{1}$ Enfermeira Residente da Marinha do Brasil. E-mail: herriet.sevilha@gmail.com

${ }^{2}$ Enfermeira da Vigilância Sanitária. E-mail: liivia paiva@yahoo.com.br

${ }^{3}$ Enfermeira. Doutora em Enfermagem. Docente da Escola de Enfermagem. Universidade de São Paulo.

Avenida Dr. Enéas de Carvalho Aguiar, 419, Sala 358. Cerqueira César. CEP 05403-000. São Paulo, SP, Brasil.

Telefone: (11) 3061-8837.E-mail: vbpoveda@usp.br 


\section{Introdução}

A infecção do sítio cirúrgico (ISC) ocupa o terceiro lugar em termos de incidência, representando entre $14 \%$ e $16 \%$ de todas as infecções nosocomiais dentro das instituições hospitalares brasileiras, merecendo destaque dadas as repercussões físicas, financeiras e sociais, que podem, inclusive, culminar com a morte do cliente acometido. Dentre estas repercussões, aquela que acomete mais diretamente a estrutura hospitalar é representada pelo aumento da estadia e, consequentemente, dos custos associados a esta; isto é, o tratamento de um paciente com infecção triplica em relação a um paciente que não a desenvolve $e^{1-3}$.

A contaminação ambiental é um dos fatores extrínsecos que influenciam diretamente a ocorrência de ISC; por isso, a prevenção da contaminação ambiental seria uma das formas de prevenção de sua ocorrência ${ }^{3}$. Assim, a limpeza de pisos, paredes e equipamentos do Centro Cirúrgico, e, também, o comportamento da equipe atuante nesse setor - como a diminuição do trânsito de pessoas, a abertura de portas, a adequada degermação das mãos e a paramentação correta - poderiam contribuir para a diminuição das taxas desta iatrogenia ${ }^{2,4}$.

$\mathrm{O}$ ar da sala cirúrgica também pode contribuir para a contaminação do ambiente. Segundo o Centers for Disease Control and Prevention (CDC), a sala operatória (SO) deve seguir alguns parâmetros para a prevenção da contaminação por partículas aéreas da área cirúrgica, como a manutenção da temperatura entre 20 e $23{ }^{\circ} \mathrm{C}$, da umidade entre 30 e $60 \%$, e, no mínimo, 15 trocas de ar por hora ${ }^{2}$.

Nesse sentido, a prevenção de ISC está relacionada ao comportamento dos profissionais que atuam no Centro Cirúrgico (CC), bem como à adequada realização de tarefas e à implementação de medidas preventivas. Portanto, este estudo pretende analisar as medidas ambientais relacionadas à prevenção de ISC em sala operatória.

\section{Objetivo geral}

- Analisar as variáveis ambientais e as medidas preventivas para evitar a ocorrência de infecção do sítio cirúrgico em pacientes submetidos a cirurgias eletivas, em um hospital filantrópico.

\section{Método}

Trata-se de estudo descritivo, observacional, transversal e com abordagem quantitativa, realizado durante a execução de procedimentos cirúrgicos em um hospital filantrópico na região do Vale do Paraíba, com enfoque nos comportamentos das Equipes Médicas e de Enfermagem.

O hospital selecionado para a condução do presente estudo atende a todas as especialidades médicas, possui 155 leitos de internação e seis salas cirúrgicas, com um fluxo de 252 cirurgias eletivas, de urgência ou emergência, por mês. Durante alguns períodos no ano, este hospital recebe estagiários de Enfermagem (nível técnico e de graduação).

Após autorização do hospital selecionado para estudo, o projeto de pesquisa foi submetido a apreciação e aprovação do Comitê de Ética em Pesquisa das Faculdades Integradas Teresa D’ Ávila (FATEA) (número de protocolo nº. 69/2011). Ao iniciarmos a investigação, os profissionais presentes nos procedimentos cirúrgicos receberam informações a respeito da investigação e assinaram o Termo de Consentimento Livre e Esclarecido (TCLE).

A coleta de dados foi realizada de novembro de 2011 a janeiro de 2012 pelos pesquisadores, em datas e horários agendados com os representantes do hospital selecionado. Para tanto, utilizou-se um instrumento de coleta de dados, que continha as seguintes informações: quantidade de participantes na cirurgia, paramentação da equipe, tempo de duração da cirurgia, temperatura e umidade da sala, número de aberturas de porta e tempo que esta permaneceu aberta, tipo de cirurgia e limpeza da sala.

Para a verificação da temperatura e da umidade ambiental, utilizou-se um termo- higrômetro digital da marca Minipa ${ }^{\circledR}$, com precisão para temperatura interna de mais ou menos $1{ }^{\circ} \mathrm{C}$ e, para umidade ambiente, de mais ou menos $8 \%$ de umidade relativa do ar, de propriedade dos pesquisadores.

Posteriormente, os dados foram analisados de forma descritiva, utilizando-se números absolutos, média e porcentagem.

\section{Resultados}

Foram analisadas 23 cirurgias de diversas especialidades, sendo sete $(30 \%)$ cirurgias ortopédicas, seguidas por cinco $(22 \%)$ cirurgias ginecológicas, quatro $(17 \%)$ cirurgias de mama, três $(13 \%)$ cirurgias vasculares, duas $(9 \%)$ cirurgias gástricas e duas $(9 \%)$ cirurgias obstétricas. Observou-se também predominância de cirurgias consideradas limpas, em número de 15 (65\%).

A duração média dos procedimentos foi de 54 minutos, considerando como início o momento da incisão cirúrgica e, como término, o último ponto na pele, com duração máxima de 140 e mínima de dez minutos.

Foram observadas as médias da temperatura e da umidade da sala operatória (SO) a cada 20 minutos de cirurgia, sendo as menores médias de temperatura e umidade do ar da sala operatória obtidas aos 140 minutos, com $21,3{ }^{\circ} \mathrm{C}$ de temperatura e $29 \%$ de umidade; ressalte-se que, em dois casos $(8,69 \%)$, não foi possível coletar os dados (Tabela 1).

Houve média de 5,69 pessoas presentes em SO por procedimento, entre os quais Cirurgião, Cirurgiões Assistentes, Anestesista, Instrumentador e circulante de sala.

Em relação a entradas e saídas de pessoas em $\mathrm{SO}$, observou-se média de 4,34 pessoas; a porta foi aberta, em média, 12,6 vezes, e permaneceu aberta, em média, por 11,89 minutos (Tabela 2). 
Quanto à utilização do avental cirúrgico, considerou-se como adequado o avental que foi vestido de maneira correta e fechado completamente na parte posterior (superior e abdominal). Dessa forma, todos os Cirurgiões usaram-no adequadamente; entre os 13 Auxiliares, nove (69\%) utilizaram adequadamente e quatro (31\%), inadequadamente; dos 29 Instrumentadores, 25 (86,2\%) estavam adequados e quatro $(13,8 \%)$, inadequados.

Considerou-se o uso de máscara como adequado, quando o dispositivo cobria totalmente a boca e o nariz, permanecendo desta forma durante todo o procedimento cirúrgico. Assim, todos os $23(100 \%)$ Cirurgiões utilizaram as máscaras adequadamente; entre os 13 Auxiliares, nove $(69,2 \%)$ usaram-na adequadamente e quatro $(30,8 \%)$, de forma inadequada; dos 25 Anestesistas, quatro (16\%) estavam adequados e 21 (84\%), inadequados; dos 32 circulantes, 14 (44\%) estavam adequados e 18 (56\%) estavam inadequados, e os $29(100 \%)$ Instrumentadores estavam adequados.
$\mathrm{Na}$ instituição onde o estudo foi realizado, disponibilizase o propé para utilização pelos profissionais, sendo que 69 sujeitos utilizaram-no; observou-se também a utilização de um calçado plástico com furos, não recomendado para Centro Cirúrgico, entre 57 profissionais; note-se que nove sujeitos utilizaram calçados fechados, de plástico, isolante elétrico, lavável, sem furos, antiderrapante e de uso exclusivo. Também se observou a utilização de sapato com cadarço, por um profissional (Tabela 3).

Para o presente estudo, considerou-se como adequado, em relação ao uso de adornos: utilização de brincos que não ultrapassassem o lóbulo da orelha e não utilização de anéis, pulseiras e correntes, em geral.

Todos os Cirurgiões e Instrumentadores usaram adequadamente adornos e, dos 13 Cirurgiões Auxiliares, $11(85 \%)$ fizeram uso adequado e dois (15\%) fizeram uso inadequado. Observamos um total de 32 circulantes; destes, 23 (72\%) fizeram uso adequado e nove (28\%), uso inadequado de adornos. Entre os 25 Anestesistas, 23 (92\%)

Tabela 1. Distribuição das médias de temperatura e umidade do ar em sala operatória. São Paulo, 2012.

\begin{tabular}{ccc}
\hline Momento de aferição & Temperatura sala operatória Média $\left({ }^{\circ} \mathrm{C}\right)$ & Umidade sala operatória Média $(\%)$ \\
\hline Incisão Cirúrgica & 26,5 & 35,5 \\
20 minutos & 23,6 & 35,2 \\
40 minutos & 22,7 & 33,0 \\
60 minutos & 23,5 & 36,1 \\
80 minutos & 22,8 & 32,2 \\
100 minutos & 22,6 & 30,0 \\
120 minutos & 22,3 & 31,3 \\
140 minutos & 21,3 & 29,0 \\
\hline
\end{tabular}

Tabela 2. Distribuição do número de pessoas presentes em sala, quantidade de entradas e saídas, abertura de portas e tempo com porta aberta em sala operatória. São Paulo, 2012.

\begin{tabular}{lccc}
\hline \multicolumn{1}{c}{ Variáveis } & Média & Número máximo & Número mínimo \\
\hline Pessoas em SO & 5,69 & 9 & 3 \\
Quantidade de entradas e saída da SO & 4,34 & 14 & 1 \\
Quantidade de vezes em que a porta foi aberta & 12,6 & 48 & 1 \\
Tempo em que a porta permaneceu aberta & 11,89 minutos & 85 minutos & 0 minuto \\
\hline
\end{tabular}

Tabela 3. Distribuição dos tipos de calçados utilizados pelos profissionais presentes em sala operatória. São Paulo, 2012.

\begin{tabular}{|c|c|c|c|c|c|c|c|c|c|}
\hline \multirow{2}{*}{ Profissional } & \multicolumn{2}{|c|}{ Propé } & \multicolumn{2}{|c|}{$\begin{array}{l}\text { Calçado fechado } \\
\text { privativo }\end{array}$} & \multicolumn{2}{|c|}{$\begin{array}{c}\text { Calçado plástico } \\
\text { com furos }\end{array}$} & \multicolumn{2}{|c|}{ Sapato com cadarço } & \multirow[t]{2}{*}{ Total } \\
\hline & $\mathrm{N}$ & $\%$ & $\mathrm{~N}$ & $\%$ & $\mathrm{~N}$ & $\%$ & $\mathrm{~N}$ & $\%$ & \\
\hline Cirurgião & 5 & 22 & 7 & 30 & 11 & 48 & & - & 23 \\
\hline Cirurgião Auxiliar & 7 & 54 & 2 & 15 & 4 & 31 & & - & 13 \\
\hline Anestesista & 15 & 60 & & & 9 & 36 & 1 & 4 & 25 \\
\hline Circulante & 11 & 34 & & & 21 & 66 & & - & 32 \\
\hline Instrumentador & 17 & 59 & & & 12 & 41 & & - & 29 \\
\hline Estagiários+Representante de empresas & 14 & 100 & & & & & & - & 14 \\
\hline
\end{tabular}


utilizaram adornos adequadamente e dois (8\%), de forma inadequada.

Foram considerados adequados esmaltes que estivessem íntegros, independentemente de sua cor. Quanto ao comprimento das unhas, estas deviam estar curtas, ou seja, que não ultrapassassem a falange distal.

Todos os Cirurgiões e Auxiliares estavam com as unhas e os esmaltes adequados. Dos 13 cirurgiões assistentes, todos $(100 \%)$ estavam com as unhas e os esmaltes adequados. Entre os 29 Instrumentadores, 27 (93\%) estavam adequados e dois (7\%) estavam inadequados. Do total de 32 circulantes, 30 (94\%) estavam adequados e dois $(6 \%)$ estavam inadequados. E entre os 25 Anestesistas observados, $22(88 \%)$ estavam adequados e três $(12 \%)$ estavam com unhas e esmaltes inadequados.

$\mathrm{O}$ uso da touca foi considerado adequado quando esta cobrisse todo o cabelo. Foi observada também a utilização de dois tipos de toucas: a descartável e a de tecido (Tabela 4).

$\mathrm{Na}$ instituição em que a pesquisa foi realizada, foi padronizado que a limpeza terminal aconteceria em dias que não houvesse cirurgias eletivas das especialidades atendidas, ocorrendo por revezamento de salas, de forma que, no mínimo, duas salas estivessem prontas para cirurgias de emergência.

Utilizava-se, para a limpeza da parede, um desinfetante à base de quaternário de amônio; para a limpeza do chão, um composto de ácido peracético, e para a limpeza de superfícies, álcool $70 \%$. Apenas um balde foi utilizado para a limpeza de todas as salas e o mesmo pano para limpeza do chão em todas as $\mathrm{SO}$.

De 23 cirurgias observadas, em 22 (96\%), houve a limpeza concorrente, sendo que, em $57 \%$, houve a limpeza do chão e da mesa cirúrgica; em $26 \%$, procedeu-se à limpeza das mesas auxiliares, da mesa cirúrgica e do chão; em $9 \%$ dos casos, apenas o chão foi submetido à limpeza; em $4 \%$ dos casos, realizou-se apenas a limpeza da mesa cirúrgica; em um procedimento (4\%), não houve a limpeza concorrente entre cirurgias.

Ressalta-se que a limpeza do mobiliário de sala foi realizada pela equipe de Enfermagem, enquanto a limpeza do chão foi realizada pela equipe de higiene e limpeza.

\section{Discussão}

Dentre as 23 cirurgias observadas no presente estudo, a maior parte delas era considerada limpa. Neste tipo de procedimento, consideram-se índices aceitáveis de infecção de 1 a $5 \%^{5}$; portanto, a ocorrência de infecções nessas cirurgias serve como um indicador de qualidade ${ }^{1}$ e um dos aspectos que podem contribuir para a ocorrência de ISC é a contaminação do ar da sala operatória por vários fatores, como roupas contaminadas, partículas vindas do ambiente externo à sala e micro-organismos do trato respiratório dos profissionais, entre outros.

Assim, quanto maior o número de pessoas dentro da $\mathrm{SO}$ ou de abertura da porta durante o ato cirúrgico, maior o risco de contaminação do $\operatorname{ar}^{6}$, o que pode, potencialmente, levar estes micro-organismos suspensos no ar ambiente até a ferida cirúrgica?

As medidas preventivas observadas, como o uso de touca, máscara e avental, e o uso de adornos e sapatos apropriados, foram adequadamente implementadas pela maior parte da equipe. Contudo, ressalta-se que, entre os que utilizaram estes recursos inadequadamente, destacam-se os membros da Equipe de Enfermagem, principalmente os circulantes de sala, e entre a Equipe Médica, os Médicos Anestesistas; portanto, membros da equipe multiprofissional que permanecem os maiores períodos de tempo no bloco operatório.

Desta maneira, se faz necessário reforçar medidas básicas de prevenção de infecção e paramentação cirúrgica, como o uso da touca ou do gorro cirúrgico, que deve cobrir todo o cabelo, de forma que não existam fios de cabelo expostos, que possam vir a se soltar durante a cirurgia ${ }^{2}$. Além disso, a utilização de máscara cobrindo nariz e a boca durante o procedimento cirúrgico previne que o paciente seja contaminado pela microbiota da orofaringe da equipe . $^{8}$.

Atualmente, as instituições de saúde, atendendo à legislação vigente, vetam o uso de adornos, tanto para a equipe de saúde quanto para a equipe de limpeza ${ }^{9}$, aspecto ressaltado por estudo que aponta uma maior contagem de bactérias gram-negativas em mãos com anéis do que em mãos sem anéis ${ }^{10}$. Além disso, sabemos que a utilização de adornos

Tabela 4. Distribuição do uso de touca descartável e de tecido pelos profissionais presentes em sala operatória. São Paulo, 2012.

\begin{tabular}{|c|c|c|c|c|c|c|c|c|}
\hline \multirow{3}{*}{ Profissional } & \multicolumn{4}{|c|}{ Touca descartável } & \multicolumn{4}{|c|}{ Touca de tecido } \\
\hline & \multicolumn{2}{|c|}{ Adequado } & \multicolumn{2}{|c|}{ Inadequado } & \multicolumn{2}{|c|}{ Adequado } & \multicolumn{2}{|c|}{ Inadequado } \\
\hline & $\mathrm{N}$ & $\%$ & $\mathrm{~N}$ & $\%$ & $\mathrm{~N}$ & $\%$ & $\mathrm{~N}$ & $\%$ \\
\hline Cirurgião & 23 & 100 & & & & & & \\
\hline Cirurgião Auxiliar & 10 & 77 & & & 10 & 23 & & \\
\hline Anestesista & 9 & 36 & 14 & 56 & 1 & 4 & 1 & 4 \\
\hline Circulante & 13 & 40 & 5 & 16 & 9 & 28 & 5 & 16 \\
\hline Instrumentador & 26 & 90 & 3 & 10 & & & & \\
\hline Estagiários+Representante de empresas & 14 & 100 & & & & & & \\
\hline
\end{tabular}


nas mãos e nos punhos, durante o ato cirúrgico, dificulta a realização da antissepsia dessas áreas ${ }^{2}$.

$\mathrm{O}$ uso de unhas compridas eleva o risco de rasgar a luva durante a operação ${ }^{2}$. Embora menos frequente no Brasil, a literatura científica aponta que o uso de unhas postiças aumenta a chance de colonização por bactérias gram-negativas e fungos; por isso, seu uso deve ser abolido. Quanto à utilização de esmaltes, seu uso deve ser com precaução, levando em consideração que esmaltes não íntegros têm uma maior chance de abrigar micro-organismos, favorecendo infecções ${ }^{10}$.

Uma das formas extrínsecas de se prevenir a contaminação dentro da sala operatória é o uso de avental cirúrgico, que forma uma barreira entre a equipe e a ferida operatória, prevenindo a contaminação. Entretanto, cabe ressaltar que a literatura científica ainda é controversa sobre as vantagens e desvantagens da utilização de aventais de uso único ou reutilizáveis, em relação à ocorrência de $\mathrm{ISC}^{11}$.

Observou-se, na presente investigação, que a maioria dos sujeitos utilizou propé e que pelo menos um terço da amostra estudada usava sapatos de plástico, com furos, o que vai contra a recomendação vigente, que estabelece o uso de sapato fechado e restrito, prevenindo assim acidentes com perfurocortantes ou contaminação por líquidos corpóreos. O uso do propé tem sido abolido, já que se sabe que sua utilização não previne a contaminação ambiental e pode, ainda, levar micro-organismos até as mãos dos funcionários, quando os mesmos tocam seus pés para retirá-lo. Dessa forma, não deve ser considerado como uma barreira de proteção ambiental, já que sua utilização previne apenas que os sapatos dos profissionais sejam sujos por sangue e outros líquidos corpóreos ${ }^{2,9,12,13}$.

Neste estudo, observou-se que a temperatura e a umidade do ar da $\mathrm{SO}$ atendiam às recomendações nacionais $\mathrm{e}$ internacionais, ou seja, estavam entre 18 e $23^{\circ} \mathrm{C}$, e a umidade entre 30 e $60 \%$. Essas recomendações respaldam-se no fato de que temperaturas elevadas em SO contribuem para a multiplicação e a proliferação de micro-organismos, e de que a baixa umidade em SO pode contribuir para a dispersão de poeira no ambiente e, por outro lado, quando alta, favorecer o crescimento de micro-organismos ${ }^{14,15}$.

Ressaltam-se, ainda, as falhas detectadas em relação às limpezas terminal e concorrente das salas de cirurgia no hospital investigado, que não atendiam as mais recentes recomendações que preveem a limpeza terminal diária das salas com utilização frequente. Observe-se que as áreas ou os equipamentos muito manuseados durante os procedimentos devem ser limpos entre pacientes (limpeza concorrente), entre os quais se destacam: a mesa cirúrgica, os equipamentos de anestesia, o suporte de soro e qualquer equipamento manipulado ao longo do procedimento, como cadeiras, mesas e focos, entre outros ${ }^{15,16}$.

Considerando-se a higienização da SO uma responsabilidade partilhada entre a Equipe de Enfermagem e a de higiene e limpeza da instituição, reforça-se a necessidade de educação continuada e supervisão direta dos procedimentos executados por estes profíssionais.

Assim, diversos aspectos presentes na prática diária podem favorecer a ocorrência de ISC; portanto, a Equipe de Enfermagem deve estar atenta aos diversos riscos ambientais e, para tanto, é necessário investir na educação dos profissionais envolvidos na assistência direta e indireta ao paciente em unidades de centro cirúrgico ${ }^{17}$, cabendo à Enfermeira o papel de protagonismo como agente disseminador e avaliador destas práticas

\section{Conclusão}

Ao final deste estudo, conclui-se que o valor médio da temperatura e da umidade relativa do ar variou dentro dos valores preconizados, exceto pela temperatura do momento da incisão cirúrgica.

A porta da sala cirúrgica foi aberta, em média, 12,6 vezes, permanecendo aberta por 11,89 minutos, em média.

Durante o ato cirúrgico, houve uma média de 5,69 pessoas em sala. Quanto ao uso de adornos, do avental cirúrgico, do sapato, da máscara e de touca/gorro, os mesmos foram utilizados de forma inadequada, de maneira mais frequente entre os circulantes de sala e Anestesistas. Ou seja: as categorias profissionais que permanecem por maior período de tempo no bloco cirúrgico são as que menos aderiram às medidas preventivas de infecção hospitalar.

Dessa forma, reforça-se a importância de fortalecer a adesão a estas práticas entre as Equipes de Enfermagem e Médicas, por meio da implementação de atividades de educação continuada e de monitoramento das medidas preventivas ambientais e comportamentais.

\section{Referências}

1. Santos MLG, Teixeira RR, Diogo A Filho. [Infecção do sítio cirúrgico em pacientes adultos submetidos a cirurgias limpas e contaminadas em hospital universitário brasileiro]. Arq Gastroenterol. 2010;47(4):383-7. PMid:21225150.

2. Mangram AJ, Horan TC, Pearson ML, Silver LC, Jarvis WR, Committee THICP, and the Hospital Infection Control Practices Advisory Committee. Guideline for prevention of surgical site infection, 1999. Infect Control Hosp Epidemiol. 1999;20(4):250-78, quiz 279-80. http://dx.doi.org/10.1086/501620. PMid:10219875.

3. Ercole FF, Franco LMC, Macieira TGR, Wenceslau LCC, Resende HIN, Chianca TCM. Risco para infecção de sitio cirúrgico em pacientes submetido a cirurgias ortopédicas. Rev Lat Am Enfermagem. 2011;19(6):1362-8. http://dx.doi.org/10.1590/ S0104-11692011000600012.

4. Brasil. Agência Nacional de Vigilância Sanitária. Higienização das mãos em serviços de saúde. Brasília: Anvisa; 2007 [acesso em 2012 Maio 20]. Disponível em: http://bvsms.saude.gov.br/ bvs/publicacoes/higienizacao_maos.pdf.

5. São Paulo. Secretaria de Estado da Saúde. Coordenadoria de Controle de Doenças. Centro de Vigilância Epidemiológica "Prof. Alexandre Vranjac". Divisão de Infecção Hospitalar. Manual de orientações e critérios diagnósticos: sistema de vigilância 
epidemiológica das infecções hospitalares do Estado de São Paulo. São Paulo: Secretária de Estado da Saúde; 2012 [acesso em 2012 Nov 22]. Disponível em: http://www.prefeitura.sp.gov.br/cidade/ secretarias/upload/chamadas/manual_criterios_diagnostico_ih hospital_geral_2012_1331926138.pdf

6. Dolinger EJ, Brito DV, Souza GM, Melo GB, Gontijo Filho PP. Contaminação do ar em salas cirúrgicas durante cirurgias de artroplastias total de quadril e joelho, hemiartroplastias e osteossínteses no centro cirúrgico de um hospital Brasileiro. Rev Soc Bras Med Trop. 2010;43(5):584-7. http://dx.doi.org/10.1590/ S0037-86822010000500023. PMid:21085875

7. Smith EB, Raphael IJ, Maltenfort MG, Honsawek S, Dolan K, Younkins EA. The effect of laminar air flow and door openings on operating room contamination. J Arthroplasty. 2013;28(9):1482-5. http://dx.doi.org/10.1016/j.arth.2013.06.012. PMid:23890828

8. Barbosa MH, Martini MMG, Teixeira JBA. Utilização de máscara facial cirúrgica descartável no ambiente cirúrgico. Rev Eletr Enf. 2009;11(2):275-9.

9. Brasil. Norma Regulamentadora 32. Segurança e saúde no trabalho em serviços de saúde, de 16 de novembro de 2005. Diário Oficial da República Federativa do Brasil; Brasília; 2005 [acesso em 2012 Maio 20]. Disponível em: http://portal.mte.gov.br/data/files/8A7C 812D36A280000138812EAFCE19E1/NR-32\%20(atualizada\%20 2011).pdf.

10. Leão MTCA. NR 32 e os adornos em estabelecimentos de saúde. Prática Hospitalar. 2007;52:44-5.
11. McHugh SM, Corrigan MA, Hill ADK, Humphreys H. Surgical attire, practices and their perception in the prevention of surgical site infection. Surgeon. 2014;12(1):47-52. http://dx.doi. org/10.1016/j.surge.2013.10.006. PMid:24268928

12. Barreto RASS, Rocha-Vilefort LO, Souza ACS, Prado-Palos MA, Barbosa MA, Borges VPFN. Processo de limpeza da sala operatória: risco à saúde do usuário e trabalhador. Rev Eletr Enf. 2011;13(2):269-75. http://dx.doi.org/10.5216/ree.v13i2.9191.

13. Braswell ML, Spruce L. Implementing AORN recommended practices for surgical attire. AORN J. 2012;95(1):122-37, quiz 13840. http://dx.doi.org/10.1016/j.aorn.2011.10.017. PMid:22201576

14. Association of Perioperative Registered Nurses - AORN. Recommended practices for sterilization. In: Association of Perioperative Registered Nurses - AORN. Perioperative standards and recommended practices. Denver: AORN; 2013. p. 513-40.. http://dx.doi.org/10.6015/psrp.12.01.e1.

15. Associação Brasileira de Enfermeiros de Centro Cirúrgico, Recuperação Anestésica e Centro de Material e Esterilização SOBECC. Práticas recomendadas SOBECC. 6. ed. São Paulo: SOBECC; 2013.

16. Allen G. Implementing AORN recommended practices for environmental cleaning. AORN J. 2014;99(5):570-9, quiz 580-2. http://dx.doi.org/10.1016/j.aorn.2014.01.023. PMid:24766919

17. Madeira MZA, Santana RAP, Santos AMR, Moura ECC. Prevenção de infecção hospitalar pela equipe cirúrgica de um hospital de ensino. Revista SOBECC. 2013;17(1):35-44. 